\title{
La consideración de la publicidad sexista en función del contexto y de las características personales. Estudio empírico entre estudiantes de publicidad
}

\author{
Marián NAVARRO-BELTRÁ ${ }^{1}$ \\ Marta MARTÍN LLAGUNO ${ }^{2}$ \\ Universidad de Alicante
}

\begin{abstract}
RESUMEN:
La regulación del sexismo publicitario se ha reconsiderado recientemente en diversas normativas públicas. No obstante, su valoración no está exenta de subjetividad. Este trabajo evalúa la influencia de los factores sociodemográficos y contextuales así como la relación de la personalidad y de los valores en la estimación del sexismo publicitario. A través de un diseño empírico cuasi experimental en el que, después del visionado de spots en situaciones diversas, 190 estudiantes rellenan una serie de cuestionarios, se concluye que ni el sexo ni el contexto de la recepción influyen significativamente en la valoración del sexismo publicitario, a su vez, la personalidad del receptor no se relaciona de forma significativa con esta evaluación, aunque sí lo hacen los valores.
\end{abstract}

PALABRAS CLAVE: Estudio de recepción; publicidad; género; publicidad sexista; legislación.

TITLE: Consideration of sexist advertising based on context and personal characteristics. Empirical study between advertising students

\begin{abstract}
The regulation of sexist advertising has been reconsidered recently in various public policies. However, their assessment is not free of subjectivity. This paper evaluates the influence of sociodemographic and contextual factors and the relationship of personality and values in the estimation of sexism advertising. Through a empirical quasi-experimental design in which, after viewing spots in different situations, 190 students filled a series of questionnaires, it is concluded that neither sex nor the context of the reception significantly influence the valuation of sexist advertising, turn, the personality of the receiver is not significantly associated with this assessment, although if the values.
\end{abstract}

KEYWORDS: Reception studies; advertising; gender; sexist advertising; legislation.

\section{Políticas públicas de género y medios de comunicación. El escenario en España}

Desde el punto de vista político, aunque el sexismo es un tema de preocupación desde las más elementales normas democráticas de alcance mundial -Derechos del

1 Becaria FPI por la Generalitat Valenciana en el Departamento de Comunicación y Psicología Social de la Universidad de Alicante. Correo electrónico: marian.navarro@ua.es

2 Catedrática de Universidad del área de Comunicación Audiovisual y Publicidad de la Universidad de Alicante. Correo electrónico: marta.martin@ua.es 
Niño, Derechos Humanos, etc. $-^{3}$, lo cierto es que, en los últimos años, se ha vuelto a retomar con fuerza en las normativas públicas, de forma que se han exigido remodelaciones legislativas con implicaciones en los discursos mediáticos.

La razón de esta trabazón es que, en general, se considera que los mass media poseen influencia sobre la población como agentes de socialización y que son capaces de reproducir y de mantener la ideología tradicional del género ${ }^{4}$. En concreto, los medios de comunicación juegan un papel esencial «en los procesos de construcción y difusión de las imágenes, identidades y relaciones de género» ${ }^{5}$. De esta forma, junto a la familia y a la escuela, nos enseñan a ser diferentes en función de nuestro sexo. Así, desde que nacemos, estamos inmersos en un proceso de socialización que consagra un «modelo femenino» $\mathrm{y}$ un «modelo masculino» ${ }^{6}$.

En este contexto, la publicidad puede llegar a convertirse en un instrumento capaz de influir en la sociedad, al confirmar (o no) los roles que el sujeto ha adquirido durante su vida ${ }^{7}$ y ofrecer valores culturales ${ }^{8}$ capaces de reforzar (o de amortiguar) los estereotipos de género".

Pese a existir abundante legislación que contempla por estas razones el respeto de la imagen de la mujer en publicidad, entre otras cuestiones y según algunos autores como

3 MARTín, M.C. y MASSó, H. (1996): Aplicación y desarrollo curricular de la igualdad de oportunidades para ambos sexos. Propuesta didáctica para el primer ciclo de Educación Secundaria Obligatoria, Madrid, Ministerio de Educación y Ciencia, http://ntic.educacion.es/w3//recursos/secundaria/transversales/index.htm. Web visitada el 06-10-2011, 1.

4 VAlladares, B. (2004-2005): «La maternidad y los medios masivos de comunicación. Un análisis de artículos periodísticos y propaganda comercial en Costa Rica», Diálogos: Revista Electrónica de Historia, 5 (1-2), http://historia.fcs.ucr.ac.cr/articulos/esp-genero/4parte/CAP22Blanca.htm. Web visitada el 04$10-2011$.

5 ESPINAR, E. (2006): «Imágenes y estereotipos de género en la programación y en la publicidad infantil. Análisis cuantitativo», Revista Latina de Comunicación Social, 61, $\mathrm{http}: / /$ www.revistalatinacs.org/200614EspinarRuiz.htm. Web visitada el 04-10-2011.

6 De los Ríos, M.J. y MarTíneZ, J. (1997): «La mujer en los medios de comunicación», Comunicar: Revista Científica Iberoamericana de Comunicación y Educación, 5 (9), 97-104,

$\mathrm{http} / / /$ www.revistacomunicar.com/index.php?contenido=detalles\&numero=9\&articulo=09-1997-14. Web visitada el 06-10-2011, 97.

7 Royo, M., Miquel, M.J. y CAPLLIURE, E.M. (2006): «Advertising content as a socialization agent: Potential reinforcement of gender stereotypes», Esic Market, 125, 81-134, http://www.esic.es/documentos/revistas/esicmk/070118_140611_I.pdf. Web visitada el 06-10-2011, 85.

8 KALlinY, M. y GeNTRY, L. (2007): «Cultural values reflected in Arab and American television advertising», Journal of Current Issues and Research in Advertising, 29 (1), 15-32,

$\mathrm{http}: / /$ scholarsmine.mst.edu/post_prints/pdf/KallinyGentry_09007dcc8055d2f9.pdf. Web visitada el 06-10$2011,17$.

9 Royo, M., AldÁs, J., KÜSTER, I. y VILA, N. (2005): «Roles de género y sexismo en la publicidad de las revistas españolas: Un análisis de las tres últimas décadas del siglo XX», Comunicación y Sociedad: Revista de la Facultad de Comunicación (Universidad de Navarra), 18 (1), 113-152,

http://www.unav.es/fcom/comunicacionysociedad/es/resumen.php?art_id=69. Web visitada el 06-10-2011, 114. 
Balaguer ${ }^{10}$, estas normativas son constantemente ignoradas por parte de la industria publicitaria debido a la dificultad «que conlleva la defensa jurídica de estos derechos».

El principal problema de las propuestas legales relacionadas con la discriminación por sexo en la comunicación comercial parece ser su aplicabilidad. En efecto, en ciertas -por no decir en la mayoría de- ocasiones, resulta realmente complejo implementarlas.

La ambigüedad terminológica se erige como el elemento fundamental de esta dificultad. Así, Camps ${ }^{11}$ señala la complejidad existente para utilizar conceptos objetivables, pues «la ley, porque es general, tiene que ser ambigua; está sujeta a aplicaciones distintas según sea la sensibilidad de los demandante o de los jueces»; es decir, las normativas públicas no pueden delimitar los términos relacionados con el sexismo publicitario, ya que sus usos y costumbres varían a lo largo del tiempo.

La dificultad que supone medir el grado de sexismo en los manifiestos publicitarios a través de las definiciones legales, especialmente cuando se considera que los estereotipos de género pueden coadyuvan a generar violencia machista ha sido, en este sentido, puesta de manifiesto con anterioridad ${ }^{12}$.

La preocupación por el sexismo publicitario queda reflejada por primera vez en la legislación en 1988 a través de la Ley General de Publicidad ${ }^{13}$. Así, se estableció como ilícita la comunicación comercial «que atente contra la dignidad de la persona o vulnere los valores y derechos reconocidos en la Constitución, especialmente en lo que se refiere a la infancia, la juventud y la mujer». Balaguer ${ }^{14}$ considera que esta definición es «meramente enunciativa, y no especifica nada acerca de cuándo un anunciante incurre en una lesión a los derechos fundamentales, y a qué derechos en concreto» se refiere. Este problema de ambigüedad terminológica y de falta de claridad parece no haberse resuelto en el marco de las nuevas normas legislativas nacidas recientemente.

En el año 2004 se promulga la Ley Integral Contra la Violencia de Género ${ }^{15}$ que afecta a los medios de comunicación por considerar que éstos pueden contribuir a fomentar la violencia de machista. Esta normativa modifica la Ley General de Publicidad al introducir los dos supuestos básicos de publicidad ilícita relacionados con el género, que tienen en cuenta la presentación vejatoria de las mujeres:

10 Balaguer, M.L. (2008): «Género y regulación de la publicidad en el ordenamiento jurídico. La imagen de la mujer», Revista Latina de Comunicación Social, 63, 382-391. doi: 10.4185/RLCS-63-2008775-382-391, 382.

11 CAMPS, V. (1998): El siglo de las mujeres, Madrid, Cátedra, 1998, 124-126.

12 Navarro-Beltrá, M. y Martín Llaguno, M (2010): «La publicidad sexista según las leyes integrales de violencia de género. Estudio empírico en España y Argentina», II Congreso Internacional Latina de Comunicación Social, La Laguna.

13 Ley 34/1988, de 11 noviembre, General de Publicidad, BOE, 15 de noviembre de 1988, 274, http://www.boe.es/diario_boe/txt.php?id=BOE-A-1988-26156. Web visitada el 06-10-2011, 32465.

14 Balaguer (2008), op. cit., 385.

15 Ley Orgánica 1/2004, de 28 de diciembre, de Medidas de Protección Integral contra la Violencia de Género, BOE, 29 de diciembre de 2004, 313, http://www.boe.es/diario_boe/txt.php?id=BOE-A-200421760. Web visitada el 06-10-2011. 
a) «Bien utilizando particular y directamente su cuerpo o partes del mismo como mero objeto desvinculado del producto que se pretende promocionar»;

b) «Bien su imagen asociada a comportamientos estereotipados que vulneren los fundamentos de nuestro ordenamiento coadyuvando a generar» violencia de género ${ }^{16}$.

Si bien el primer precepto es fácilmente valorable, con referencia al segundo precepto, Rodríguez y Tato ${ }^{17}$ entienden que el legislador considera que se trata de forma vejatoria a la mujer a) cuando ésta aparece desempeñando una labor vinculada tradicionalmente con las féminas y (no o) b) del mensaje publicitario se desprende la idea de que esta tarea es exclusiva y propia de la población femenina.

No obstante, Rodríguez ${ }^{18}$ también establece que no se precisa el contenido de la publicidad ilícita relacionada con la utilización de estereotipos asociados a la imagen de la mujer que coadyuven a generar violencia de género. La indefinición permite que el término estereotipo sea interpretado de muy diversas formas. Según Tato, y llevado al extremo, incluso podría considerarse que «impide la representación publicitaria de la mujer desempeñando cualquier actividad que, según una escala de valores afortunadamente superada, estuviese tradicionalmente reservado para las mujeres» ${ }^{19}$.

Coincidimos con otros autores al pensar que la modificación del artículo 3a de Ley General de Publicidad, por parte de la Ley Integral de Violencia de Género, no añade ninguna novedad importante con referencia a la redacción anterior ${ }^{20}$. Así, Tato ${ }^{21}$ estima que dicha modificación ha sido innecesaria, poco eficaz y compleja, ya que el escrito original, bien considerado, ya condenaba la publicidad sexista y discriminatoria más hiriente y grave. Sin embargo, sólo una interpretación excesivamente laxa y flexible del actual artículo podría incluir los supuestos más graves e hirientes de publicidad sexista.

En definitiva, la legislación española que regula la imagen de la mujer en la publicidad conlleva una compleja interpretación ${ }^{22}$, situación que deriva en su dificultad para ser aplicada.

Junto a estas circunstancias, se observa una escasez de investigaciones sobre el sexismo publicitario centradas en el proceso de la recepción mediática. De esta forma, los análisis bibliográficos indican que, entre 1988 y 2010 únicamente se han publicado cuatro artículos, y ninguna tesis doctoral, relacionadas con esta temática ${ }^{23}$. Si se tiene

16 Ibidem, 42183.

17 RoDrígueZ, A. (2008): «Aportaciones de la Ley de medidas contra la violencia de género a la prohibición de publicidad discriminatoria», Revista de Derecho de la Competencia y la Distribución, 2, 145-160, 155; TATO, A. (2006): «Violencia de género y publicidad», Revista Autocontrol, 107, 1-6, 4-5.

18 RODRÍGUEZ (2008), op. cit., 155.

19 TATO (2006), op. cit., 4.

20 RODRÍGUEZ (2008), op. cit., 155.

21 TATO (2006), op. cit., 2-5.

22 Ibidem, 3.

23 NAvarro-Beltrá, M. y Martín Llaguno, M. (2011): «El sexismo publicitario: delimitación de conceptos e indicadores de género. Estudio empírico de la producción científica», Pensar la Publicidad. Revista Internacional de Investigaciones Publicitarias, 5 (1), 51-73. doi:

10.5209/rev_PEPU.2011.v5.n1.3692, 62-63. 
en cuenta que la trasmisión y la formación de valores en los seres humanos se produce a través de la interacción de varios factores -la escuela, los amigos, la familia, la sociedad en general y los medios de comunicación de forma destacada $-{ }^{24}$ y que los estudios de audiencias prestan atención al contexto de los receptores, ya que éstos crean «los marcos idóneos dentro de los cuales los mensajes adquirirán sentido» ${ }^{25}$, este hecho no es baladí, dado el valor de la interpretación en la consideración del «sexismo». No en vano, y en este sentido, nos podemos encontrar ante escenarios de disparidad sobre aquello que el legislador y la población consideran como «publicidad ilícita».

\section{Objetivos}

El objetivo general del trabajo es explorar las actitudes de la audiencia hacia las representaciones de género mostradas en la publicidad y su consonancia o disonancia con las de las instituciones encargadas de juzgar la existencia o inexistencia de sexismo publicitario. A su vez, se pretende valorar la influencia o la relación de las características personales y del contexto de recepción en esas actitudes.

De forma específica se persigue:

a) Averiguar la concordancia de los juicios sobre el sexismo publicitario entre los jueces que dilucidan la presencia o ausencia de discriminación por sexo en la comunicación comercial y una audiencia de estudiantes de publicidad;

b) Examinar la influencia del sexo y la relación de la personalidad y de la escala de valores del receptor en la percepción del sexismo publicitario;

c) Sondear la repercusión del contexto de visionado sobre la percepción del sexismo, en concreto, valorar si influye ver la publicidad en solitario o en compañía;

d) Estudiar si la influencia del contexto de visionado depende del sexo del participante, del sexo del acompañante o de la relación que ambos mantienen.

\section{Método}

\subsection{Metodología}

Con el fin de acometer los objetivos planteados, entre los meses de marzo y abril de 2011, se realizó una investigación empírica cuasi experimental. En concreto, se recurrió a la técnica de los cuestionarios para analizar la percepción de la población sobre

24 JiMÉNEZ, J.R. (1997): «La educación y valores y los medios de comunicación», Comunicar: Revista Científica Iberoamericana de Comunicación y Educación, 5 (9), 15-22,

$\mathrm{http}: / /$ www.revistacomunicar.com/index.php?contenido=detalles\&numero=9\&articulo=09-1997-04. Web visitada el 06-10-2011, 16.

25 Corominas, M. (2001): «Los estudios de recepción», Lecciones del Portal, http://www.portalcomunicacion.com/uploads/pdf/4_esp.pdf. Web visitada el 04-10-2011, 3. 
las representaciones de mujeres y hombres en los manifiestos publicitarios y sus posibles relaciones e influencias.

\subsection{Participantes}

Debido a que se trata de un estudio piloto, ya que a mejor conocimiento no existen en España investigaciones con objetivos similares centradas en alumnos de publicidad -futuros encargados de elaborar la comunicación comercial y, por tanto, de decidir las representaciones de género mostradas en los anuncios-, se recurrió a una muestra de conveniencia (no probabilística) ${ }^{26}$. En concreto, la población a analizar estuvo formada por la totalidad de las personas matriculadas en el Grado de Publicidad y Relaciones Públicas de la Universidad de Alicante del curso académico 2010-2011.

Se solicitó la participación, de forma voluntaria y no evaluable, de los 241 alumnos inscritos en estos estudios, finalmente, decidieron cooperar en la investigación 190. En concreto, intervinieron 79 hombres $(41,6 \%)$ y 111 mujeres $(58,4 \%)$, con una media de edad de 20 años.

A su vez, se pidió a los estudiantes que buscaran la colaboración de un acompañante, de esta forma, y en función de la situación asignada por grupo de prácticas, los alumnos recurrieron al padre en 23 ocasiones $(12,1 \%)$, a la madre en $33(14,4 \%)$, a un colega del mismo sexo en $48(25,3 \%)$, a un colega del sexo opuesto en $36(18,9 \%)$ y a un estudiante de publicidad y relaciones públicas en 50 (26,3\%). El 54,2\% de los acompañantes fueron mujeres $(\mathrm{n}=103)$ y el $45,8 \%$ varones $(\mathrm{n}=87)$.

\subsection{Estímulos}

Los dos spots que debían visionar los participantes en el estudio antes de cumplimentar los cuestionarios fueron seleccionados por haber sido emitidos por televisión ${ }^{27}$ después de que la Ley General de Publicidad fuera modificada por la Ley Integral de Violencia de Género al introducir los dos supuestos de publicidad ilícita vinculados con la población femenina y por haber sido calificados de sexistas por la audiencia, ya que el Observatorio de la Imagen de las Mujeres -anteriormente conocido como el Observatorio de la Publicidad Sexista- recibió diversas quejas acerca de estos manifiestos publicitarios. Además, estos anuncios de forma individual o las campañas a las que pertenecen de forma global, fueron considerados como publicidad sexista por el Instituto de la Mujer y por el Instituto Andaluz de la Mujer, dos de los entes legitimados por

26 Wimmer, R.D. y Dominick, J.R. (1996): La investigación cientifica de los medios de comunicación, Barcelona, Bosch casa editorial, 67.

27 La televisión es capaz de educar, incluso, a través de sus contenidos publicitarios. En EsPINAR, E. (2007): «Las raíces socioculturales de la violencia de género», Escuela Abierta: Revista de Investigación Educativa, 10, 23-48, http://www.ceuandalucia.com/escuelaabierta/pdf/articulos_ea10/02espinar.pdf. Web visitada el 06-10-2011, 34. 
las normativas públicas para solicitar la cesación o la prohibición de la publicación de la comunicación comercial sexista, aunque sin capacidad para imponer sanciones.

Cada anuncio podría hacer referencia a uno de los supuestos contemplados como publicidad sexista por la legislación española. En uno de ellos, de la marca Axe, aparece el cuerpo de la mujer desvinculado del producto promocionado ${ }^{28} \mathrm{y}$, en el otro, que publicita el canal de ciencia ficción Syfy, se muestra a una mujer realizando las labores del hogar mientras su marido e hijo ven la televisión ${ }^{29}$.

\subsection{Procedimiento}

El primer paso para poder efectuar el presente trabajo consistió en la elaboración de dos cuestionarios. Pese a que los instrumentos de recogida de información fueron creados ex profeso para esta investigación, como se verá mas adelante, fueron el producto obtenido tras adaptar anteriores investigaciones.

En segundo lugar, los participantes cumplimentaron los cuestionarios de forma online a través de una página web mantenida por la Universidad de Alicante. Para ello, los estudiantes fueron informados de los objetivos de la investigación y se les aseguró la confidencialidad de los datos aportados. De esta forma, no se solicitó ninguna referencia que pudiera identificar a los sujetos, por tanto, para poder relacionar las dos herramientas de recogida de información, a cada alumno se le asignó un código de forma aleatoria.

Los participantes, para poder cumplimentar los dos cuestionarios, debían visionar los spots seleccionados de forma individual y rellenar a continuación el cuestionario uno, a partir de ese momento, disponían de un mes para volver a ver los mismos anuncios, pero en esta ocasión en compañía y en función de la situación asignada, y realizar el cuestionario dos.

Finalmente, para poder analizar la información recolectada, se realizó una base de datos en el programa informático SPSS versión 15. En concreto, se comprobó la fiabilidad $^{30}$ y se efectuaron análisis factoriales ${ }^{31}$ de las escalas utilizadas, se recurrió al test de la $t$ de Student para muestras independientes ${ }^{32}$ con la finalidad observar la influencia

28 El anuncio se encuentra disponible en Youtube, http://www.youtube.com/watch?v=izIr4Bbki7o\&feature=related. Web visitada el 04-10-2011.

$29 \mathrm{El}$ anuncio se encuentra disponible en Youtube, http://www.youtube.com/watch?v=nCipnJiWC8. Web visitada 04-10-2011.

30 Con el propósito de valorar la consistencia interna de las escalas se recurrió al coeficiente Alfa de Cronbach.

31 Para obtener la estructura factorial de las escalas utilizadas en los cuestionarios se recurrió al análisis factorial exploratorio de componentes principales con rotación Varimax. Además, se suprimieron de los factores resultantes los ítems con valores absolutos menores o iguales que 0,3 .

32 Se utilizó esta prueba paramétrica debido a que se cumplieron los criterios de distribución normal y homogeneidad de varianzas. Para poder establecer si las influencias que se producen son estadísticamente significativas, habitualmente se consideran los criterios $\mathrm{p}<0,05$ e intervalo de confianza del 95\%. En PÉRTEGA, S. y PITA, S. (2001): «Métodos paramétricos para la comparación de dos medias. $\mathrm{t}$ de Student», Cadernos de Atención Primaria, 8 (1), 37-41, 
del sexo en las actitudes hacia el rol de género mostrado en los anuncios, se realizaron correlaciones bivariadas ${ }^{33}$ para observar la relación existente entre estas actitudes y los valores neosexistas y la personalidad de los participantes, se empleó la prueba t para muestras relacionadas ${ }^{34}$ con el propósito de comprobar las diferencias entre el primer y el segundo visionados y se observaron diversos estadísticos descriptivos ${ }^{35}$ para complementar la información anterior.

\subsection{Instrumentos de recogida de información}

El cuestionario uno, además de una serie de datos sociodemográficos sobre los alumnos, incluía una medida de personalidad, específicamente, la escala de autoevaluaciones centrales (Core Self-Evaluation Scale), a su vez, incorporaba la escala de valores neosexistas (Neosexim Scale) y la de actitudes hacia los roles en la publicidad (Attitudes Toward Role Portrayals). Por su parte, el cuestionario dos también recolectaba datos sociodemográficos, aunque en esta ocasión sobre el alumno y su acompa-

http://www.fisterra.com/mbe/investiga/t_student/t_student2.pdf. Web visitada el 06-10-2011, 37-38.

A su vez, hay que tener en cuenta el valor de $t$, así, según su tabla de distribución, el nivel de significación para el test de dos colas cuando los grados de libertad superan la cifra 120, en este caso hay 188, es 1,960. En FisHER, R.A. y YATES, F. (1938): Statistical tables for biological, agricultural and medical research, Edinburgh, Oliver and Boyd, 1974,

http://digital.library.adelaide.edu.au/coll/special/fisher/stat_tab.pdf. Web visitada el 06-10-2011, 46.

Por tanto, para considerar que la relación existente no depende exclusivamente del azar, la significación bilateral debe ser menor que 0,05 y el valor de $t$ debe ser menor o igual que $-1,960$ o mayor o igual que $+1,960$. En WIMMER y DOMINICK (1996), op. cit., 258.

Por su parte, el tamaño del efecto únicamente se calculó en el caso de encontrar diferencias estadísticamente significativas, para ello, se usó la fórmula de Cohen, que consiste en dividir la diferencia de medias de ambos grupos por las desviaciones típicas de las puntuaciones. En COHEN, J. (1969): Statistical power analysis for the behavioral sciences, Hillsdale, Lawrence Erlbaum Associates, 1988, 20; MORALES, P. (2007): «Estadística aplicada a las ciencias sociales. El contraste de medias»,

www.upcomillas.es/personal/peter/estadisticabasica/ContrasteDeMedias.pdf. Web visitada el 04-10-2011, 31-33.

33 Se recurrió al coeficiente de correlación $r$ de Pearson y la prueba de significación escogida fue bilateral. En el caso de existir diferencias estadísticamente significativas se calculó el coeficiente de determinación $\left(\mathrm{CD}=\mathrm{r}^{2}\right)$, que multiplicado por 100 indica el porcentaje de varianza que una variable explica de otra con la que posee relación. En WIMMER y DOMINICK (1996), op. cit., 267.

${ }_{34}$ Se utilizó una prueba paramétrica porque la distribución obtenida fue normal. En el caso de encontrar diferencias estadísticamente significativas $(\mathrm{p}<0,05$, intervalo de confianza del $95 \%$ y valor de $\mathrm{t}$ en función de los grados de libertad), el tamaño del efecto se calculó dividiendo la diferencia de las medias del primer y del segundo visionado por la desviación típica obtenida tras ver el anuncio en compañía. En MoRALES, P. (2011): «Estadística aplicada a las ciencias sociales. El tamaño del efecto (effect size): Análisis complementarios al contraste de medias»,

www.upcomillas.es/personal/peter/investigacion/Tama\%F1oDelEfecto.pdf. Web visitada el 04-10-2011, 19.

35 Se observó la media, la desviación típica y los valores máximos y mínimos (extraídos a través de las frecuencias) de las preguntas referentes a la percepción sobre la influencia del contexto y de los factores obtenidos en la escala de actitudes. 
ñante, e incorporaba, además, la escala de actitudes y una serie de preguntas sobre la influencia del contexto situacional ${ }^{36}$.

\subsubsection{Medidas}

- Core Self-Evaluations Scale (CSES)

La escala de autoevaluaciones centrales fue creada por Judge, Erez, Bono y Thoresen en el año $2003^{37}$ con el propósito de valorar la personalidad de los sujetos en función de cuatro aspectos esenciales: autoestima, autoeficacia, locus de control y neuroticismo. Esta medida está formada por 12 ítems, seis negativos y seis positivos, con cinco posibles opciones de respuesta, donde 1 es totalmente en desacuerdo y 5 totalmente de acuerdo ${ }^{38}$. Esta dimensión es frecuentemente utilizada por la psicología organizacional y ha sido empleada con anterioridad en estudios vinculados con la comunicación $^{39}$.

Debido a que la escala original está redactada en lengua inglesa y recolecta datos de empleados y estudiantes, se decidió utilizar la adaptación de Hernández Ruiz -que posee una adecuada consistencia interna ${ }^{40}$ - ya que emplea el castellano y modifica algunos de sus ítems para adecuarlos a una muestra formada únicamente por alumnado universitario.

En nuestro estudio, el análisis de fiabilidad de la escala de autoevaluaciones centrales según el Alfa de Cronbach también demostró una adecuada consistencia interna $(\alpha=0,720)$. Por su parte, el análisis factorial (ver Fig. 1) proporcionó una estructura de tres factores que explicaban el $49,182 \%$ de la varianza $(\mathrm{KMO}=0,746)$. El primer factor, evaluaciones negativas, estaba compuesto por cuatro ítems y agrupaba el $26,324 \%$ varianza, el segundo factor, evaluaciones positivas, poseía seis elementos y contemplaba el $12,376 \%$ de la varianza y, el tercer factor, locus de control, con dos ítems explicaba el $10,482 \%$ de la varianza.

36 ¿Tus percepciones, sensaciones y/o valoraciones sobre los spots han sido distintas tras ver el anuncio solo/a y en compañía? ¿Has percibido diferencias en el grado de sexismo según el contexto? ¿En qué situación has considerado que los anuncios eran más sexista? e Indica brevemente las sensaciones, percepciones y valoraciones de los spots en función del contexto situacional.

37 Judge, T.A., EREZ, A., BONO, J.E. y THORESEN, C.J. (2003): «The core self-evaluations scale: Development of a measure», Personnel Psychology, 56 (2), 303-331.

doi: 10.1111/j.1744-6570.2003.tb00152.x, 303-304.

38 Ibidem, 314-315.

39 Ver, por ejemplo, HeRnáNDEZ RuIZ, A. (2008): Expectativas de vida familiar y laboral de una muestra de estudiantes de Publicidad y Relaciones Públicas, Tesis doctoral, Alicante, Universidad de Alicante, http:/rua.ua.es/dspace/handle/10045/9599. Web visitada el 06-10-2011; MARTín LLAGUNO, M. y HERNÁNDEz RuIz, A. (2010): «Radiografía de los docentes en publicidad en España. Un estudio psicolaboral con enfoque de género», Pensar la Publicidad. Revista Internacional de Investigaciones Publicitarias, 4 (2), 25-50, http://revistas.ucm.es/index.php/PEPU/article/view/PEPU1010220025A/15017. Web visitada el 06-10-2011.

40 HERNÁNDEZ RUIZ (2008), op. cit., 187 y 195-196. 
Fig. 1: distribución de factores para la escala de autoevaluaciones centrales ${ }^{a}$

\begin{tabular}{|c|c|c|c|}
\hline \multirow[b]{2}{*}{ Ítem } & \multicolumn{3}{|c|}{ Componente } \\
\hline & $\begin{array}{c}\text { Factor 1: eva- } \\
\text { luaciones nega- } \\
\text { tivas }\end{array}$ & $\begin{array}{c}\text { Factor 2: } \\
\text { evaluaciones } \\
\text { positivas }\end{array}$ & $\begin{array}{c}\text { Factor 3: } \\
\text { locus de } \\
\text { control }\end{array}$ \\
\hline $\begin{array}{l}\text { 12. (Invertido). A veces las cosas parecen } \\
\text { desoladoras y sin esperanzas para mí }\end{array}$ & 0,798 & & \\
\hline $\begin{array}{l}\text { 2. (Invertido). A veces me siento depri- } \\
\text { mido/a }\end{array}$ & 0,762 & & \\
\hline $\begin{array}{l}\text { 4. (Invertido). Si suspendo me siento un/a } \\
\text { inútil }\end{array}$ & 0,700 & & \\
\hline $\begin{array}{l}\text { 8. (Invertido). Tengo dudas sobre mis } \\
\text { aptitudes }\end{array}$ & 0,464 & 0,364 & \\
\hline $\begin{array}{l}\text { 1. Conseguiré el éxito que merezco en la } \\
\text { vida }\end{array}$ & & 0,754 & \\
\hline 5. Termino las tareas con éxito & & 0,554 & \\
\hline 7. Estoy satisfecho/a conmigo mismo/a & 0,462 & 0,528 & \\
\hline 3. Cuando lo intento tengo éxito & & 0,522 & $-0,385$ \\
\hline 9. Decido lo que sucederá en mi vida & & 0,505 & \\
\hline 11. Soy capaz de afrontar problemas & & 0,372 & \\
\hline $\begin{array}{l}\text { 6. (Invertido). No tengo el control sobre } \\
\text { mis estudios }\end{array}$ & & & 0,813 \\
\hline $\begin{array}{l}\text { 10. (Invertido). No tengo el control sobre } \\
\text { mis estudios en la universidad }\end{array}$ & & & 0,783 \\
\hline
\end{tabular}

Método de extracción: Análisis de componentes principales.

Método de rotación: Normalización Varimax con Kaiser.

${ }^{a}$ La rotación ha convergido en 5 iteraciones.

\section{- Neosexim Scale}

Esta medida fue creada por Tougas, Brown, Beaton y Joly en 1995 con el propósito de valorar nuevas modalidades de sexismo ${ }^{41}$. Se trata de una escala de 11 ítems con siete posibles opciones de respuesta, donde 1 es muy en desacuerdo y 7 muy de acuer$\mathrm{do}^{42}$. En el presente estudio se utilizó la versión validada y adaptada a castellano por Moya y Expósito ${ }^{43}$ que demostró unas características psicométricas adecuadas y una consistencia interna apropiada.

En nuestra muestra, el análisis de fiabilidad de la escala de valores neosexistas, según el Alfa de Cronbach, también demostró una adecuada consistencia interna $(\alpha=0,775)$. Por su parte, tras realizar el análisis factorial (ver Fig. 2) se obtuvieron tres

41 LAMEIRAS, M., RoDríGUEZ, Y. y CALADO, M. (2006): «El neosexismo y diferencias de género en las metas de trabajo», Revista de Psicología Social, 21 (3), 223-228. doi: 10.1174/021347406778538212, 225.

42 MoyA, M. y Expósito, F. (2001): «Nuevas formas, viejos intereses: Neosexismo en varones españoles», Psicothema, 13 (4), 643-649, http://www.psicothema.com/pdf/492.pdf. Web visitada el 06-10-2011, 645.

43 Ibidem, 645 y 647. 
factores que agrupaban el $57,416 \%$ de la varianza $(\mathrm{KMO}=0,813)$ : el primer factor, logros inmerecidos, explicaba el $33,617 \%$ de la varianza y contaba con cinco ítems; el segundo factor, no hay discriminación, poseía el $12,502 \%$ de la varianza e incluía cuatro elementos y; el tercer factor, presión social, englobaba el $11,297 \%$ de la varianza y estaba formado por dos ítems.

Fig. 2: distribución de factores para la escala de neosexismo ${ }^{a}$

\begin{tabular}{|c|c|c|c|}
\hline & \multicolumn{3}{|c|}{ Componente } \\
\hline & $\begin{array}{c}\text { Factor 1: } \\
\text { logros } \\
\text { inmerecidos } \\
\end{array}$ & $\begin{array}{l}\text { Factor 2: no } \\
\text { hay discri- } \\
\text { minación }\end{array}$ & $\begin{array}{l}\text { Factor 3: } \\
\text { presión so- } \\
\text { cial } \\
\end{array}$ \\
\hline $\begin{array}{l}\text { 3. Las mujeres no deberían meterse donde no } \\
\text { se las quiere }\end{array}$ & 0,794 & & \\
\hline 5 Es difícil trabajar para un jefe que sea mujer & 0,777 & & \\
\hline $\begin{array}{l}\text { 8. Las universidades se equivocan al admitir a } \\
\text { las mujeres en carreras muy costosas tales } \\
\text { como Medicina, porque de hecho, un gran } \\
\text { número de ellas abandonarán después de unos } \\
\text { años para cuidar a sus hijos }\end{array}$ & 0,720 & & \\
\hline $\begin{array}{l}\text { 7. Desde hace unos pocos años, las mujeres } \\
\text { han conseguido del gobierno más de lo que se } \\
\text { merecen }\end{array}$ & 0,703 & & \\
\hline $\begin{array}{l}\text { 4. Las mujeres progresarían más siendo pa- } \\
\text { cientes en lugar de presionar tanto para lograr } \\
\text { cambios }\end{array}$ & 0,580 & 0,488 & \\
\hline $\begin{array}{l}\text { 2. (Invertido). El actual sistema de empleo } \\
\text { laboral es injusto para las mujeres }\end{array}$ & & 0,733 & \\
\hline $\begin{array}{l}\text { 1. La discriminación contra la mujer en el } \\
\text { terreno laboral no es un problema grave en } \\
\text { España }\end{array}$ & & 0,650 & \\
\hline $\begin{array}{l}\text { 6. Las demandas de las mujeres en términos } \\
\text { de igualdad entre los sexos son simplemente } \\
\text { exageradas }\end{array}$ & 0,490 & 0,623 & \\
\hline $\begin{array}{l}\text { 11. (Invertido). En un sistema de empleo } \\
\text { justo, hombres y mujeres deberían ser consi- } \\
\text { derados iguales }\end{array}$ & & 0,317 & \\
\hline $\begin{array}{l}\text { 9. En orden a no parecer sexistas, muchos } \\
\text { hombres se inclinan a sobreproteger a las } \\
\text { mujeres }\end{array}$ & & & 0,798 \\
\hline $\begin{array}{l}\text { 10. Debido a la presión social, muchas empre- } \\
\text { sas contratan a mujeres poco cualificadas }\end{array}$ & & & 0,795 \\
\hline
\end{tabular}

Método de extracción: Análisis de componentes principales.

Método de rotación: Normalización Varimax con Kaiser.

${ }^{a}$ La rotación ha convergido en 5 iteraciones. 


\section{- Attitudes Toward Role Portrayals}

En 1977 Lundstrom y Sciglimpaglia fueron los encargados de contrastar, originalmente, esta escala ${ }^{44}$ que posee 12 ítems y siete puntos como posibles opciones de respuesta, donde 1 es muy en desacuerdo y 7 muy de acuerdo ${ }^{45}$. Sin embargo, para la realización de esta investigación se utilizó la traducción efectuada por Bigné y Cruz ${ }^{46}$ en el año 2000.

Esta medida fue concebida con el propósito de evaluar las actitudes hacia la representación de los roles en la publicidad de forma general, no obstante, en este estudio se emplea con la finalidad de valorar estas actitudes hacia dos anuncios concretos, calificados anteriormente como sexistas. Por tanto, fue necesario adaptar la medida y prescindir de aquellos ítems que hicieran referencia explícita a la publicidad de manera general, en concreto, se eliminaron los elementos 12, «Overall, I believe that the portrayal of women in advertising is changing for the better», y 10, «I'm more sensitive to the portrayal of women in advertising than I used to be» ${ }^{47}$.

El análisis de fiabilidad, según el Alfa de Cronbach, de los 10 ítems considerados demostró una adecuada consistencia interna $(\alpha=0,606)$. Por su parte, el análisis factorial $^{48}$ (ver Fig. 3) puso de manifiesto la existencia de una estructura de dos factores que explicaban el $58,214 \%$ de la varianza $(\mathrm{KMO}=0,806)$. El primer factor, los anuncios visionados son sexistas, estaba compuesto por cinco elementos y el $36,079 \%$ de la varianza. El segundo factor, los anuncios visionados no son sexistas, también estaba formado por cinco ítems, pero en esta ocasión agrupaba el 22,135\% de la varianza.

Fig. 3: distribución de factores para la escala de actitudes hacia los roles de género en la publicidad ${ }^{a}$

\begin{tabular}{|c|c|c|}
\hline \multirow{2}{*}{ Ítem } & \multicolumn{2}{|c|}{ Componente } \\
\hline & $\begin{array}{c}\text { Factor 1: los anuncios } \\
\text { son sexistas }\end{array}$ & $\begin{array}{c}\text { Factor 2: los anuncios no } \\
\text { son sexistas }\end{array}$ \\
\hline $\begin{array}{l}\text { 9. Los anuncios sugieren que el lugar } \\
\text { de la mujer está en el hogar }\end{array}$ & 0,846 & \\
\hline $\begin{array}{l}\text { 8. Los anuncios sugieren que las muje- } \\
\text { res no hacen cosas importantes }\end{array}$ & 0,831 & \\
\hline $\begin{array}{l}\text { 4. Los anuncios tratan a las mujeres } \\
\text { prácticamente como objetos sexuales }\end{array}$ & 0,786 & \\
\hline
\end{tabular}

44 BignÉ, J.E. y CRUZ, S. (2000): «Actitudes hacia los roles de género en la publicidad. Efectos sobre la imagen de empresa y la intención de compra», Cuadernos de Economía y Dirección de la Empresa, 6, 165-186, http://www.acede.org/fotos/pdf/art159_6_01.pdf. Web visitada el 06-10-2011, 175.

45 LundSTROM, W.J. y Sciglimpaglia, D. (1977): «Sex role portrayals in advertising. Are women and men critical of the way they are shown in ads, and how does this affect their attitudes toward products?», Journal of Marketing, 41 (3), 72-79, 73-75.

46 BignÉ y CRUZ (2000), op. cit.

47 LundSTROM y SCIGLimPaglia (1977), op. cit., 73.

48 Esta medida fue recolectada en dos ocasiones, no obstante, el análisis factorial y la fiabilidad que aquí se muestra corresponde a la primera toma de datos, es decir, tras ver los anuncios en solitario. 


\begin{tabular}{|lcc|}
\hline \multicolumn{1}{|c|}{ Ítem } & \multicolumn{2}{c|}{ Componente } \\
\cline { 2 - 3 } & $\begin{array}{c}\text { Factor 1: los anuncios } \\
\text { son sexistas }\end{array}$ & $\begin{array}{c}\text { Factor 2: los anuncios no } \\
\text { son sexistas }\end{array}$ \\
\hline $\begin{array}{l}\text { 11. Considero que la representación de } \\
\text { las mujeres en la publicidad es ofensiva }\end{array}$ & 0,756 & \\
\hline $\begin{array}{l}\text { 2. Los anuncios sugieren que las muje- } \\
\text { res dependen de los hombres }\end{array}$ & 0,593 & 0,796 \\
\hline $\begin{array}{l}\text { 3. Los anuncios que veo muestran a los } \\
\text { hombres como realmente son }\end{array}$ & 0,740 \\
\hline $\begin{array}{l}\text { 7. Los anuncios que veo representan } \\
\text { con precisión a los hombres en la ma- } \\
\text { yoría de sus actividades cotidianas }\end{array}$ & & 0,676 \\
\hline $\begin{array}{l}\text { 5. Los anuncios que veo representan } \\
\text { con precisión a las mujeres en la mayo- } \\
\text { ría de sus actividades cotidianas }\end{array}$ & & 0,644 \\
\hline $\begin{array}{l}\text { 1. Los anuncios que veo muestran a las } \\
\text { mujeres como realmente son }\end{array}$ & $-0,479$ & 0,544 \\
\hline $\begin{array}{l}\text { 6. Los anuncios sugieren que las muje- } \\
\text { res toman decisiones importantes }\end{array}$ & $-0,427$ & \\
\hline
\end{tabular}

Método de extracción: Análisis de componentes principales.

Método de rotación: Normalización Varimax con Kaiser.

${ }^{a}$ La rotación ha convergido en 3 iteraciones.

\section{Resultados}

\subsection{Consideración de la publicidad sexista}

$\mathrm{Al}$ analizar si las actitudes de los sujetos hacia los roles de género mostrados en los anuncios se asemejan a la delimitación de la publicidad sexista hecha por los jueces encargados de valorar la existencia o inexistencia de discriminación por sexo en la comunicación comercial, se puede afirmar que los encuestados tienen una tendencia ligeramente superior a percibir discriminación por sexo en los spots visionados que a no hacerlo. De esta forma, coinciden con la interpretación del Instituto de la Mujer y del Instituto Andaluz de la Mujer que considera estos manifiestos como sexistas.

Sin embargo, al tener en cuenta la media de los dos factores que componen la escala de actitudes hacia los roles de mujeres hombres en la publicidad, se pone de manifiesto la existencia de unas puntuaciones muy cercanas al valor neutro (4). Así, la media para el factor referente a los anuncios son sexistas es de 4,443 $(\mathrm{DT}=1,214)$ tras el visionado en solitario y de 4,579 (DT=1,152) tras el visionado en compañía. Por su parte, al considerar los spots como no son sexistas la media alcanza los valores $3,196(\mathrm{DT}=1,020)$ y 3,309 (DT=1,001) tras ver los anuncios en solitario y en compañía respectivamente. 


\subsection{Influencia del sexo en las actitudes hacia el rol de género mostrado en los anuncios}

Como se puede observar en la Fig. 4, la prueba paramétrica t de Student muestra que únicamente existen diferencias estadísticamente significativas por sexo en las actitudes hacia el rol de género en los spots en el caso de haber visto los anuncios en compañía y percibir sexismo en ellos $(\mathrm{p}=0,023, \mathrm{t}=2,292)$. En concreto, la Fig. 5 establece que las mujeres observan $(\mathrm{M}=4,739$, $\mathrm{DT}=1,074)$, en mayor medida que los hombres $(\mathrm{M}=4,354, \mathrm{DT}=1,225)$, que los manifiestos publicitarios son discriminatorios hacia la población femenina. No obstante, según Cohen $^{49}$, el tamaño del efecto es pequeño $(d=0,337)$.

Fig. 4: prueba t para muestras independientes en función del sexo en la escala de actitudes hacia los roles en la publicidad

\begin{tabular}{|c|c|c|c|c|c|}
\hline \multirow[b]{2}{*}{ Factor } & \multicolumn{5}{|c|}{ Prueba T para la igualdad de medias } \\
\hline & $\mathbf{t}$ & gl & $\begin{array}{c}\text { Sig. } \\
\text { (bilateral) (p) }\end{array}$ & $\begin{array}{l}\text { Diferencia } \\
\text { de medias }\end{array}$ & $\begin{array}{l}\text { Error típ. de la } \\
\text { diferencia }\end{array}$ \\
\hline $\begin{array}{l}\text { Anuncios sexistas } \\
\text { (solitario) }\end{array}$ & 1,806 & 188 & 0,073 & 0,321 & 0,178 \\
\hline $\begin{array}{l}\text { Anuncios no sexistas } \\
\text { (solitario) }\end{array}$ & 1,049 & 188 & 0,296 & 0,157 & 0,15 \\
\hline $\begin{array}{l}\text { Anuncios sexistas } \\
\text { (compañía) }\end{array}$ & 2,292 & 188 & 0,023 & 0,384 & 0,168 \\
\hline $\begin{array}{l}\text { Anuncios no sexistas } \\
\text { (compañía) }\end{array}$ & 0,506 & 188 & 0,614 & 0,075 & 0,148 \\
\hline
\end{tabular}

Fig. 5: estadísticos de grupo de la escala de actitudes hacia los roles en la publicidad por sexo

\begin{tabular}{|llcccc|}
\hline \multicolumn{1}{|c}{ Factor } & $\begin{array}{c}\text { Sexo del } \\
\text { alumno }\end{array}$ & N & $\begin{array}{c}\text { Media } \\
\text { (M) }\end{array}$ & $\begin{array}{c}\text { Desviación típ. } \\
\text { (DT) }\end{array}$ & $\begin{array}{c}\text { Error típ. de } \\
\text { la media }\end{array}$ \\
\hline $\begin{array}{l}\text { Anuncios sexistas } \\
\text { solitario) }\end{array}$ & Mujer & 111 & 4,577 & 1,158 & 0,11 \\
\hline Anuncios no sexistas (en & Mombre & 79 & 4,256 & 1,273 & 0,143 \\
\hline $\begin{array}{lllll}\text { solitario) } \\
\text { Anuncios sexistas }\end{array}$ & Hombre & 79 & 3,104 & 1,019 & 0,097 \\
compañía) & Mujer & 111 & 4,739 & 1,074 & 0,115 \\
\hline & Hombre & 79 & 4,354 & 1,225 & 0,138 \\
\hline
\end{tabular}

49 COHEN (1969), op. cit., 25-26. 


\begin{tabular}{|llcccc|}
\hline \multicolumn{1}{|c}{ Factor } & $\begin{array}{c}\text { Sexo del } \\
\text { alumno }\end{array}$ & N & $\begin{array}{c}\text { Media } \\
\text { (M) }\end{array}$ & $\begin{array}{c}\text { Desviación típ. } \\
\text { (DT) }\end{array}$ & $\begin{array}{c}\text { Error típ. de } \\
\text { la media }\end{array}$ \\
\hline $\begin{array}{l}\text { Anuncios no sexistas (en } \\
\text { compañía) }\end{array}$ & $\begin{array}{l}\text { Mujer } \\
\text { Hombre }\end{array}$ & 111 & 3,34 & 1,053 & 0,1 \\
\hline
\end{tabular}

\subsection{Relación existente entre la personalidad y los valores neosexistas en las actitudes hacia el rol de género mostrado en las anuncios}

La Fig. 6, que muestra las correlaciones bivariadas, establece que no suelen existir relaciones estadísticamente significativas entre la personalidad de los individuos y sus actitudes hacia los roles de género mostrados en los anuncios. De esta forma, únicamente se puede observar una correlación significativa negativa -a medida que aumenta la puntuación en una de las variables disminuye en la otra- entre los factores referentes a considerar sexistas los anuncios vistos en compañía y las evaluaciones negativas $(\mathrm{p}=0,038)$, no obstante, el tamaño del efecto existente es pequeño ${ }^{50}(\mathrm{r}=-0,151)$. Así, el coeficiente de determinación alcanza el valor 0,023 , ya que la varianza explicada de una de las variables por la otra es 2,3\%.

Por su parte, es más frecuente observar relaciones estadísticamente significativas entre los valores neosexistas y la consideración del sexismo publicitario. En concreto, a mayor creencia de que los logros obtenidos por las mujeres son inmerecidos aumenta la percepción de que los anuncios visionados en solitario no son sexistas $(\mathrm{p}=0,013)$, sin embargo, el tamaño del efecto es pequeño ${ }^{51}(\mathrm{r}=0,179)$. De esta forma, una de las variables explica el $3,2 \%$ de la varianza de la otra variable $(C D=0,032)$.

A su vez, se produce una relación negativa con referencia al factor que establece que no existe discriminación hacia las mujeres y la consideración de que los spots visionados son sexistas. En el caso de ver los anuncios en solitario $(\mathrm{p}=0,000)$ el tamaño del efecto encontrado es pequeño ${ }^{52}(r=-0,284)$. Igualmente, el tamaño del efecto hallado $(r=-0,211)$ al ver los anuncios en compañía $(\mathrm{p}=0,003)$ es pequeño ${ }^{53}$, así, el coeficiente de determinación alcanza el valor 0,044 , lo que supone que una variable explica el $4,4 \%$ de la varianza de la otra.

50 Ibídem, 79-80.

51 Ibídem, 79-80.

52 Ibídem, 79-80.

53 Ibidem, 79-80. 
Fig. 6: relación existente entre la personalidad y el neosexismo frente a las actitudes hacia los roles mostrados en la publicidad

\begin{tabular}{|c|c|c|c|c|c|}
\hline Factor & Datos & $\begin{array}{l}\text { Anuncios } \\
\text { sexistas } \\
\text { (solitario) }\end{array}$ & $\begin{array}{l}\text { Anuncios no } \\
\text { sexistas (soli- } \\
\text { tario) }\end{array}$ & $\begin{array}{c}\text { Anuncios } \\
\text { sexistas } \\
\text { (compañía) }\end{array}$ & $\begin{array}{c}\text { Anuncios no } \\
\text { sexistas } \\
\text { (compañía) }\end{array}$ \\
\hline \multirow{3}{*}{$\begin{array}{l}\text { Evaluacio- } \\
\text { nes negati- } \\
\text { vas }\end{array}$} & $\begin{array}{l}\text { Correlación de } \\
\text { Pearson (r) }\end{array}$ & $-0,135$ & $-0,041$ & $-0,151\left(^{*}\right)$ & $-0,077$ \\
\hline & $\begin{array}{l}\text { Sig. (bilateral) } \\
\text { (p) }\end{array}$ & 0,063 & 0,573 & 0,038 & 0,292 \\
\hline & $\mathrm{N}$ & 190 & 190 & 190 & 190 \\
\hline \multirow{3}{*}{$\begin{array}{l}\text { Evaluacio- } \\
\text { nes positi- } \\
\text { vas }\end{array}$} & $\begin{array}{l}\text { Correlación de } \\
\text { Pearson (r) }\end{array}$ & 0,006 & $-0,049$ & 0,078 & $-0,007$ \\
\hline & $\begin{array}{l}\text { Sig. (bilateral) } \\
\text { (p) }\end{array}$ & 0,934 & 0,500 & 0,287 & 0,923 \\
\hline & $\mathrm{N}$ & 190 & 190 & 190 & 190 \\
\hline \multirow{3}{*}{$\begin{array}{l}\text { Locus de } \\
\text { control }\end{array}$} & $\begin{array}{l}\text { Correlación de } \\
\text { Pearson (r) }\end{array}$ & $-0,008$ & $-0,066$ & 0,071 & 0,031 \\
\hline & $\begin{array}{l}\text { Sig. (bilateral) } \\
\text { (p) }\end{array}$ & 0,910 & 0,363 & 0,334 & 0,667 \\
\hline & $\mathrm{N}$ & 190 & 190 & 190 & 190 \\
\hline \multirow{3}{*}{$\begin{array}{l}\text { Logros } \\
\text { inmere- } \\
\text { cidos }\end{array}$} & $\begin{array}{l}\text { Correlación de } \\
\text { Pearson (r) }\end{array}$ & $-0,089$ & $0,179(*)$ & $-0,086$ & 0,066 \\
\hline & $\begin{array}{l}\text { Sig. (bilateral) } \\
\text { (p) }\end{array}$ & 0,224 & 0,013 & 0,239 & 0,366 \\
\hline & $\mathrm{N}$ & 190 & 190 & 190 & 190 \\
\hline \multirow{3}{*}{$\begin{array}{l}\text { No hay } \\
\text { discrimi- } \\
\text { nación }\end{array}$} & $\begin{array}{l}\text { Correlación de } \\
\text { Pearson (r) }\end{array}$ & $-0,284(* *)$ & $-0,037$ & $-0,211(* *)$ & $-0,021$ \\
\hline & $\begin{array}{l}\text { Sig. (bilateral) } \\
\text { (p) }\end{array}$ & 0,000 & 0,611 & 0,003 & 0,769 \\
\hline & $\mathrm{N}$ & 190 & 190 & 190 & 190 \\
\hline \multirow{3}{*}{$\begin{array}{l}\text { Presión } \\
\text { social }\end{array}$} & $\begin{array}{l}\text { Correlación de } \\
\text { Pearson (r) }\end{array}$ & 0,126 & $-0,018$ & $-0,038$ & $-0,039$ \\
\hline & $\begin{array}{l}\text { Sig. (bilateral) } \\
\text { (p) }\end{array}$ & 0,082 & 0,803 & 0,607 & 0,593 \\
\hline & $\mathrm{N}$ & 190 & 190 & 190 & 190 \\
\hline
\end{tabular}

**. La correlación es significativa al nivel 0,01 (bilateral).

*. La correlación es significativa al nivel 0,05 (bilateral). 


\subsection{Variación de las actitudes hacia el rol de género mostrado en los anuncios en función del contexto de visionado}

La prueba t para muestras relacionadas establece que no se producen diferencias estadísticamente significativas entre ver los anuncios en solitario y en compañía para los dos factores obtenidos en la escala de actitudes (ver Fig. 7).

No obstante, la Fig. 8 señala que con el segundo visionado aumentan ligeramente las puntuaciones de ambos factores. De esta forma, la consideración acerca de que los anuncios son sexistas posee una media de $4,443(\mathrm{DT}=1,214)$ para el primer visionado y de 4,579 $(\mathrm{DT}=1,152)$ para el segundo $(\mathrm{p}=0,106, \mathrm{t}=-1,624)$, en el caso del factor que establece que los anuncios no son sexistas, la media alcanza el valor de 3,196 $(\mathrm{DT}=1,02)$ tras ver los anuncios en solitario y de 3,309 (DT=1,001) $(\mathrm{p}=0,131, \mathrm{t}=-$ 1,519) al visionar los spots en compañía.

Fig. 7: prueba t para muestras relacionadas en función de la escala de actitudes hacia los roles mostrados en la publicidad

\begin{tabular}{|c|c|c|c|c|c|c|}
\hline \multirow[b]{2}{*}{ Factor } & \multicolumn{3}{|c|}{ Diferencias relacionadas } & \multirow[b]{2}{*}{$\mathbf{t}$} & \multirow[b]{2}{*}{ gl } & \multirow[b]{2}{*}{$\begin{array}{c}\text { Sig. } \\
\text { (bilateral) } \\
\text { (p) }\end{array}$} \\
\hline & $\begin{array}{c}\text { Media } \\
\text { (M) }\end{array}$ & $\begin{array}{c}\text { Desviación } \\
\text { típ. (DT) }\end{array}$ & $\begin{array}{c}\text { Error típ. } \\
\text { de la } \\
\text { media }\end{array}$ & & & \\
\hline $\begin{array}{l}\text { Anuncios sexistas: } \\
\text { en solitario - } \\
\text { en compañía }\end{array}$ & $-0,136$ & 1,152 & 0,084 & $-1,624$ & 189 & 0,106 \\
\hline $\begin{array}{l}\text { Anuncios no sexis- } \\
\text { tas: en solitario - } \\
\text { en compañía }\end{array}$ & $-0,114$ & 1,032 & 0,075 & $-1,519$ & 189 & 0,131 \\
\hline
\end{tabular}

Fig. 8: estadísticos de la escala de actitudeshacia los roles en la publicidad

\begin{tabular}{|lcccc|}
\hline \multicolumn{1}{|c}{ Factor } & $\begin{array}{c}\text { Media } \\
\text { (M) }\end{array}$ & $\mathbf{N}$ & $\begin{array}{c}\text { Desviación } \\
\text { típ. (DT) }\end{array}$ & $\begin{array}{c}\text { Error típ. de } \\
\text { la media }\end{array}$ \\
\hline Anuncios sexistas (en solitario) & 4,443 & 190 & 1,214 & 0,088 \\
\hline Anuncios sexistas (en compañía) & 4,579 & 190 & 1,152 & 0,084 \\
\hline Anuncios no sexistas (en solitario) & 3,196 & 190 & 1,02 & 0,074 \\
\hline Anuncios no sexistas (en compañía) & 3,309 & 190 & 1,001 & 0,073 \\
\hline
\end{tabular}

Sin embargo, el 70,5\% de los alumnos encuestados afirma no haber tenido una percepción, una sensación o una valoración distinta sobre la discriminación por sexo tras 
ver los anuncios en solitario y en compañía. Así, han advertido el mismo grado de sexismo en ambas situaciones el $58,9 \%$ de los participantes.

\subsection{Diferencias por sexo y por relación alumno-acompañante en las dos medidas de la escala de actitudes hacia el rol de género mostrado en los anuncios}

La Fig. 9, que ofrece la prueba t para muestras relacionadas, pone de manifiesto que no existen diferencias estadísticamente significativas por sexo del alumno entre ver los anuncios en solitario y en compañía para los dos factores obtenidos en la escala de actitudes. De esta forma, con referencia a la consideración de que los anuncios son sexistas, las mujeres alcanzan una disimilitud en sus puntuaciones medias del primer y segundo visionado de $-0,162(\mathrm{p}=0,116, \mathrm{t}=-1,585)$ y los hombres de $-0,099(\mathrm{p}=0,487$, $\mathrm{t}=-0,699)$. En el caso de percibir que los anuncios no son sexistas, la desemejanza en las medias para las féminas es de $-0,079(\mathrm{p}=0,444, \mathrm{t}=-0,768)$ y para los varones de $0,162(\mathrm{p}=0,135, \mathrm{t}=-1,511)$.

Fig. 9: prueba t para muestras relacionadas en función de la escala de actitudes hacia los roles mostrados en la publicidad por sexo del alumno

\begin{tabular}{|c|c|c|c|c|c|c|c|}
\hline \multirow[b]{2}{*}{$\begin{array}{l}\text { Sexo del } \\
\text { alumno }\end{array}$} & \multirow[b]{2}{*}{ Factor } & \multicolumn{3}{|c|}{ Diferencias relacionadas } & \multirow[b]{2}{*}{$\mathbf{t}$} & \multirow[b]{2}{*}{ gl } & \multirow[b]{2}{*}{$\begin{array}{l}\text { Sig. } \\
\text { (bilate- } \\
\text { ral) (p) }\end{array}$} \\
\hline & & $\begin{array}{c}\text { Media } \\
\text { (M) }\end{array}$ & $\begin{array}{l}\text { Desvia- } \\
\text { ción típ. } \\
\text { (DT) }\end{array}$ & $\begin{array}{c}\text { Error típ. } \\
\text { de la } \\
\text { media }\end{array}$ & & & \\
\hline \multirow{2}{*}{ Mujer } & $\begin{array}{l}\text { Anuncios } \\
\text { sexistas: en } \\
\text { solitario - en } \\
\text { compañía }\end{array}$ & $-0,162$ & 1,078 & 0,102 & $-1,585$ & 110 & 0,116 \\
\hline & $\begin{array}{l}\text { Anuncios no } \\
\text { sexistas: en } \\
\text { solitario - en } \\
\text { compañía }\end{array}$ & $-0,079$ & 1,087 & 0,103 & $-0,768$ & 110 & 0,444 \\
\hline \multirow{2}{*}{ Hombre } & $\begin{array}{l}\text { Anuncios } \\
\text { sexistas: en } \\
\text { solitario - en } \\
\text { compañía }\end{array}$ & $-0,099$ & 1,255 & 0,141 & $-0,699$ & 78 & 0,487 \\
\hline & $\begin{array}{l}\text { Anuncios no } \\
\text { sexistas: en } \\
\text { solitario - en } \\
\text { compañía }\end{array}$ & $-0,162$ & 0,953 & 0,107 & $-1,511$ & 78 & 0,135 \\
\hline
\end{tabular}

Como establece la Fig. 10, tampoco existen diferencias estadísticamente significativas entre ver los anuncios en solitario y en compañía para los dos factores de la escala de actitudes en función del sexo del acompañante. En este sentido, al percibir sexismo en los spots considerados, las mujeres obtienen una diversidad de medias entre ambos 
visionado de $-0,194(\mathrm{p}=0,099, \mathrm{t}=-1,663)$ y los hombres de $-0,067(\mathrm{p}=0,579, \mathrm{t}=-0,558)$. Cuando los participantes no advierten discriminación por sexo, la disparidad de medias para la población femenina es de $-0,078(\mathrm{p}=0,423, \mathrm{t}=-0,804)$ y para los varones es de $0,156(\mathrm{p}=0,186, \mathrm{t}=-1,333)$.

Fig. 10: prueba t para muestras relacionadas en función de la escala de actitudes hacia los roles mostrados en la publicidad por sexo del acompañante

\begin{tabular}{|lllllllll|}
\hline \multirow{2}{*}{$\begin{array}{c}\text { Sexo del } \\
\text { acom- } \\
\text { pañante }\end{array}$} & Factor & $\begin{array}{c}\text { Media } \\
\text { (M) }\end{array}$ & $\begin{array}{c}\text { Desvia- } \\
\text { ción típ. } \\
\text { (DT) }\end{array}$ & $\begin{array}{c}\text { Error típ. } \\
\text { de la } \\
\text { media }\end{array}$ & t & gl & $\begin{array}{c}\text { Sig. } \\
\text { (bilate- } \\
\text { ral) (p) }\end{array}$ \\
\hline & $\begin{array}{l}\text { Anuncios } \\
\text { sexistas: en } \\
\text { solitario }- \text { en } \\
\text { compañía }\end{array}$ & $-0,194$ & 1,185 & 0,117 & $-1,663$ & 102 & 0,099 \\
& $\begin{array}{l}\text { Anuncios no } \\
\text { sexistas: en } \\
\text { solitario }- \text { en } \\
\text { compañía }\end{array}$ & $-0,078$ & 0,98 & 0,097 & $-0,804$ & 102 & 0,423 \\
\hline Hombre & $\begin{array}{l}\text { Anuncios } \\
\text { sexistas: en } \\
\text { solitario }- \text { en } \\
\text { compañía } \\
\text { Anuncios no } \\
\text { sexistas: en } \\
\text { solitario }- \text { en } \\
\text { compañía }\end{array}$ & $-0,067$ & 1,115 & 0,119 & $-0,558$ & 86 & 0,579 \\
\hline
\end{tabular}

Por su parte, al considerar la relación existente entre el alumno y su acompañante únicamente se producen diferencias estadísticamente significativas entre las dos medidas de la escala de actitudes cuando el encuestado percibe sexismo publicitario y ha visto los spots junto a un amigo/a del mismo $\operatorname{sexo}^{54}(\mathrm{p}=0,015, \mathrm{t}=-2,534)$. De esta forma, la desemejanza de medias entre ambos visionados alcanza el valor de $-0,446$, lo que supone percibir un mayor grado de sexismo en el segundo visionado, no obstante, el tamaño del efecto es, según Cohen ${ }^{55}$, pequeño $(\mathrm{d}=0,38)$.

54 El valor de t que establece la significación estadística para el test de dos colas, $\mathrm{p}<0,05$ y 47 grados de libertad, es 2,021. En FISHER y YATES (1938), op. cit., 46.

De esta forma, cuando $\mathrm{p}<0,05, \mathrm{t}$ debe ser menor o igual que $-2,021$ o mayor o igual que $+2,021$ para considerar que la influencia existente no depende de forma exclusiva del azar. En WIMMER y DOMINICK (1996), op. cit., p. 258.

55 COHEN (1969), op. cit., 25-26. 


\section{Conclusiones y discusión}

Los resultados hallados en el presente estudio cuasi experimental establecen que los encuestados tienen una ligera tendencia a considerar la existencia de discriminación por género en aquellos anuncios que muestran a la mujer como objeto sexual o en los que aparece realizando las tareas del hogar mientras el varón disfruta de su tiempo de ocio, dos representaciones de la población femenina que el Instituto de la Mujer y el Instituto Andaluz de la Mujer han calificado de ilícitas. Pese a la existencia de una audiencia teóricamente sensibilizada hacia el tratamiento no sexista de la mujer en la publicidad (como son los estudiantes de comunicación comercial) los y las encuestadas señalan valores próximos al 4 (neutro). Este resultado parece indicar la necesidad de reforzar la perspectiva de género en la docencia para sensibilizar a los creadores de anuncios y evitar así que las representaciones sexistas de los últimos años estén aumentando ${ }^{56}$.

En este sentido, cabe señalar que el sexo de los alumnos no suele influir en sus actitudes hacia los roles de género mostrados en la publicidad, ya que únicamente se pueden observar influencias estadísticamente significativas en el supuesto de considerar los anuncios, visionados en compañía, como sexistas. En este caso, y al igual que se afirma en otros trabajos, los varones tienden a ser menos críticos que las féminas ${ }^{57}$.

A su vez, la personalidad de los participantes no suele asociarse de manera estadísticamente significativa con sus actitudes hacia las representaciones de género en los anuncios, pues esta correlación solamente se produce entre las evaluaciones negativas y el considerar sexistas los spots vistos en compañía.

No obstante, los valores neosexistas de los encuestados sí se relacionan, de forma significativa, con sus actitudes hacia los roles de mujeres y hombres ofrecidos en la comunicación comercial. Especialmente destacable es la correlación que establece que a mayor creencia de que no existe discriminación hacia la población femenina disminuye la percepción acerca de que los anuncios visionados son sexistas, ya sean vistos en solitario o en compañía. En efecto, las personas más críticas con la discriminación social de la mujer son las más sensibles a la representación de los géneros en la publicidad $^{58}$, pues son las que suelen percibir sexismo en los anuncios visionados.

Por tanto, la presente investigación establece que el sexo de los participantes no suele influir en sus actitudes hacia el rol de género mostrado en los anuncios y que no es habitual que la personalidad se relacione de forma significativa con estas actitudes.

56 REID, A. y JONES, S.C. (2010): «The use of female sexuality in Australian alcohol advertising: Public policy implications of young adults' reactions to stereotypes», Journal of Public Affairs, 10 (1-2), 1935. doi: 10.1002/pa.339, 33 .

57 Bigné y CRUZ (2000), op. cit., 177; LundSTROM y SCIGLIMPAGLia (1977), op. cit., 75.

58 Ford, J.B., LATOUR, M.S. y LunDSTROM, W.J. (1991): «Comtemporary women's evaluation of female role portrayals in advertising», The Journal of Consumer Marketing, 8 (1), 15-28. doi: 10.1108/07363769110034901, 23. 
Sin embargo, los valores, y en concreto la apreciación de la no existencia de discriminación hacia la mujer, sí se asocian con las actitudes señaladas.

Nos preguntamos si es posible que el legislador, y los sujetos encargados de juzgar el sexismo en la publicidad, puedan ofrecer respuestas, si bien no objetivas, sí sistemáticas en el tiempo. Consideramos que es difícil, ya que los encuestados -entes activos en las futuras representaciones de género de los anuncios- establecen su apreciación del sexismo publicitario en función de sus valores y, en ocasiones, del contexto.

Es importante destacar la escasa presencia de trabajos que analizan el punto de vista de la audiencia acerca de las representaciones de género mostradas en la publicidad ${ }^{59}$, en concreto, «el consumo femenino de la imagen de las mujeres en los medios no es uno de los temas más emergentes en las investigaciones sobre género y medios» ${ }^{60}$. En otras palabras, en las últimas tres décadas apenas se ha avanzado en la investigación a cerca de la reacción de la audiencia sobre los discursos publicitarios con sesgos de género. Por tanto, cabría preguntarse, con quién y cuánto ha podido contrastar el legislador sus consideraciones de publicidad sexista.

Finalmente, cabe señalar que la principal limitación del presente trabajo hace referencia a la selección de los participantes, ya que los cuestionarios han sido cumplimentados únicamente por alumnado del Grado de Publicidad y Relaciones Públicas de la Universidad de Alicante.

Esta situación podría explicar la escasez de las desemejanzas halladas y el pequeño tamaño del efecto encontrado en las diferencias estadísticamente significativas, pues los sujetos comparten un mismo contexto sociocultural y unas mismas características personales, como la edad y el interés por la publicidad.

Pese a que consideramos que la elección de los encuestados es válida por tratarse de un estudio piloto, los resultados obtenidos son difícilmente representativos de los estudiantes de publicidad en España. Así, si se escogiera una muestra diferente, las conclusiones podrían no coincidir con las obtenidas en esta investigación.

De esta forma, una posible línea de trabajo consistiría en replicar el estudio utilizando una muestra más amplia y repetirlo también dentro de unos años para comprobar si las actitudes hacia el rol de género varían a lo largo del tiempo.

\section{Referencias bibliográficas}

BALAGUER, M.L. (2008): «Género y regulación de la publicidad en el ordenamiento jurídico. La imagen de la mujer», Revista Latina de Comunicación Social, 63, 382-391. doi: 10.4185/RLCS-63-2008-775-382-391.

59 Navarro-Beltrá, M. y Martín Llaguno, M. (2011), op, cit., 62-63.

60 GARCíA, N. y MARTíNEZ, L. (2009): «La representación positiva de la imagen de las mujeres en los medios», Comunicar: Revista Científica Iberoamericana de Comunicación y Educación, 16 (32), 209-214. doi: 10.3916/c32-2009-03-002, 211. 
BigNÉ, J.E. y CRUZ, S. (2000): «Actitudes hacia los roles de género en la publicidad. Efectos sobre la imagen de empresa y la intención de compra», Cuadernos de Economía y Dirección de la Empresa, 6, 165-186, http://www.acede.org/fotos/pdf/art159_6_01.pdf. Web visitada el 06-10-2011.

CAMPS, V. (1998): El siglo de las mujeres, Madrid, Cátedra, 1998.

COHEN, J. (1969): Statistical power analysis for the behavioral sciences, Hillsdale, Lawrence Erlbaum Associates, 1988.

COROMINAS, M. (2001): «Los estudios de recepción», Lecciones del Portal, http://www.portalcomunicacion.com/uploads/pdf/4_esp.pdf. Web visitada el 04-10-2011.

De los Ríos, M.J. y MARTíneZ, J. (1997): «La mujer en los medios de comunicación», Comunicar: Revista Científica Iberoamericana de Comunicación y Educación, 5 (9), 97-104, http://www.revistacomunicar.com/index.php?contenido $=$ detalles\&numero=9\&articulo $=09$ 1997-14. Web visitada el 06-10-2011.

ESPINAR, E. (2007): «Las raíces socioculturales de la violencia de género», Escuela Abierta: Revista de Investigación Educativa, 10, 23-48,

http://www.ceuandalucia.com/escuelaabierta/pdf/articulos_ea10/02espinar.pdf. Web visitada el 06-10-2011.

- (2006): «Imágenes y estereotipos de género en la programación y en la publicidad infantil. Análisis cuantitativo», Revista Latina de Comunicación Social, 61, http://www.revistalatinacs.org/200614EspinarRuiz.htm. Web visitada el 04-10-2011.

FISHER, R.A. y YATES, F. (1938): Statistical tables for biological, agricultural and medical research, Edinburgh, Oliver and Boyd, 1974, http://digital.library.adelaide.edu.au/coll/special/fisher/stat_tab.pdf. Web visitada el 06-102011.

FORD, J.B., LATOUR, M.S. y LUNDSTROM, W.J. (1991): «Comtemporary women's evaluation of female role portrayals in advertising», The Journal of Consumer Marketing, 8 (1), 15-28. doi: 10.1108/07363769110034901.

GARCÍA, N. y MARTíNEZ, L. (2009): «La representación positiva de la imagen de las mujeres en los medios», Comunicar: Revista Científica Iberoamericana de Comunicación y Educación, 16 (32), 209-214. doi: 10.3916/c32-2009-03-002.

HERNÁNDEZ RUIZ, A. (2008): Expectativas de vida familiar y laboral de una muestra de estudiantes de Publicidad y Relaciones Públicas, Tesis doctoral, Alicante, Universidad de Alicante, http://rua.ua.es/dspace/handle/10045/9599. Web visitada el 06-10-2011.

JIMÉNEZ, J.R. (1997): «La educación y valores y los medios de comunicación», Comunicar: Revista Científica Iberoamericana de Comunicación y Educación, 5 (9), 15-22, $\mathrm{http}: / /$ www.revistacomunicar.com/index.php?contenido=detalles\&numero $=9 \&$ articulo $=09$ 1997-04. Web visitada el 06-10-2011.

Judge, T.A., EREZ, A., BonO, J.E. y THORESEN, C.J. (2003): «The core self-evaluations scale: Development of a measure», Personnel Psychology, 56 (2), 303-331. doi: 10.1111/j.17446570.2003.tb00152.x.

KALLINY, M. y GENTRY, L. (2007): «Cultural values reflected in Arab and American television advertising», Journal of Current Issues and Research in Advertising, 29 (1), 15-32, http://scholarsmine.mst.edu/post_prints/pdf/KallinyGentry_09007dcc8055d2f9.pdf. Web visitada el 06-10-2011. 
LAMEIRAS, M., RodríguEZ, Y. y CALADO, M. (2006): «El neosexismo y diferencias de género en las metas de trabajo», Revista de Psicología Social, 21 (3), 223-228. doi: 10.1174/021347406778538212.

Ley 34/1988, de 11 noviembre, General de Publicidad, BOE, 15 de noviembre de 1988, 274, http://www.boe.es/diario_boe/txt.php?id=BOE-A-1988-26156. Web visitada el 06-10-2011.

Ley Orgánica 1/2004, de 28 de diciembre, de Medidas de Protección Integral contra la Violencia de Género, BOE, 29 de diciembre de 2004, 313, http://www.boe.es/diario_boe/txt.php?id=BOE-A-2004-21760. Web visitada el 06-10-2011.

LUNDSTROM, W.J. y SCIGLIMPAGLIA, D. (1977): «Sex role portrayals in advertising. Are women and men critical of the way they are shown in ads, and how does this affect their attitudes toward products?», Journal of Marketing, 41 (3), 72-79.

MARTín LlagunO, M. y HeRnÁDEZ RuIZ, A. (2010): «Radiografía de los docentes en publicidad en España. Un estudio psicolaboral con enfoque de género», Pensar la Publicidad. Revista Internacional de Investigaciones Publicitarias, 4 (2), 25-50,

http://revistas.ucm.es/index.php/PEPU/article/view/PEPU1010220025A/15017. Web visitada el 06-10-2011.

MARTín, M.C. y MASSÓ, H. (1996): Aplicación y desarrollo curricular de la igualdad de oportunidades para ambos sexos. Propuesta didáctica para el primer ciclo de Educación Secundaria Obligatoria, Madrid, Ministerio de Educación y Ciencia, http://ntic.educacion.es/w3//recursos/secundaria/transversales/index.htm. Web visitada el 0610-2011.

MoraleS, P. (2011): «Estadística aplicada a las ciencias sociales. El tamaño del efecto (effect size): Análisis complementarios al contraste de medias»,

www.upcomillas.es/personal/peter/investigacion/Tama\%F1oDelEfecto.pdf. Web visitada el 04-10-2011.

- (2007): «Estadística aplicada a las ciencias sociales. El contraste de medias», www.upcomillas.es/personal/peter/estadisticabasica/ContrasteDeMedias.pdf. Web visitada el 04-10-2011.

MoYA, M. y EXPÓSITO, F. (2001): «Nuevas formas, viejos intereses: Neosexismo en varones españoles», Psicothema, 13 (4), 643-649, http://www.psicothema.com/pdf/492.pdf. Web visitada el 06-10-2011.

NAVARro-BeltrÁ, M. y Martín Llaguno, M (2010): «La publicidad sexista según las leyes integrales de violencia de género. Estudio empírico en España y Argentina», II Congreso Internacional Latina de Comunicación Social, La Laguna.

- (2011): «El sexismo publicitario: delimitación de conceptos e indicadores de género. Estudio empírico de la producción científica», Pensar la Publicidad. Revista Internacional de Investigaciones Publicitarias, 5 (1), 51-73. doi: 10.5209/rev_PEPU.2011.v5.n1.3692.

PÉRTEGA, S. y PITA, S. (2001): «Métodos paramétricos para la comparación de dos medias. $t$ de Student», Cadernos de Atención Primaria, 8 (1), 37-41, $\mathrm{http}: / / \mathrm{www}$. fisterra.com/mbe/investiga/t_student/t_student2.pdf. Web visitada el 06-102011.

REID, A. y JONES, S.C. (2010): «The use of female sexuality in Australian alcohol advertising: Public policy implications of young adults' reactions to stereotypes», Journal of Public Affairs, 10 (1-2), 19-35. doi: 10.1002/pa.339. 
RoDRíGUEZ, A. (2008): «Aportaciones de la Ley de medidas contra la violencia de género a la prohibición de publicidad discriminatoria», Revista de Derecho de la Competencia y la Distribución, 2, 145-160.

ROYO, M., ALDÁs, J., KÜSTER, I. y VILA, N. (2005): «Roles de género y sexismo en la publicidad de las revistas españolas: Un análisis de las tres últimas décadas del siglo XX», Comunicación y Sociedad: Revista de la Facultad de Comunicación (Universidad de Navarra), 18 (1), 113-152, http://www.unav.es/fcom/comunicacionysociedad/es/resumen.php?art_id=69. Web visitada el 06-10-2011.

Royo, M., MiQuel, M.J. y CAPLliure, E.M. (2006): «Advertising content as a socialization agent: Potential reinforcement of gender stereotypes», Esic Market, 125, 81-134, http://www.esic.es/documentos/revistas/esicmk/070118_140611_I.pdf. Web visitada el 06$10-2011$.

TAto, A. (2006): «Violencia de género y publicidad», Revista Autocontrol, 107, 1-6.

VALLADARES, B. (2004-2005): «La maternidad y los medios masivos de comunicación. Un análisis de artículos periodísticos y propaganda comercial en Costa Rica», Diálogos: Revista Electrónica de Historia, 5 (1-2),

http://historia.fcs.ucr.ac.cr/articulos/esp-genero/4parte/CAP22Blanca.htm. Web visitada el 04-10-2011.

WIMMER, R.D. y DOMINICK, J.R. (1996): La investigación cientifica de los medios de comunicación, Barcelona, Bosch casa editorial. 